\title{
Commentary: Effectiveness of A Multimodal Training Programme to Improve General Practitioners' Burnout, Job Satisfaction and Psychological Well-Being
}

\author{
Carles Barcons Comellas ${ }^{1,2 *}$ \\ 'Clinical Psychologist, Hospital Sagrat Cor, Serveis de Salut Mental Martorell, Spain \\ ${ }^{2}$ PhD student, Department of Psychiatry and Forensic Medicine, Universitat Autònoma de Barcelona, Spain
}

Article Info

\section{Article Notes}

Received: December 5, 2019

Accepted: January 8, 2020

\section{${ }^{*}$ Correspondence:}

Carles Barcons Comellas, Clinical Psychologist, Hospital Sagrat Cor, Serveis de Salut Mental Martorell \& PhD student, Department of Psychiatry and Forensic Medicine, Universitat Autònoma de Barcelona, Spain; Email: barcons@hotmail.com.

(c) 2020 Barcons $C$. This article is distributed under the terms of the Creative Commons Attribution 4.0 International License.
It is necessary to improve GP's psychological resources and capacities that may assist them, especially when treating mentalhealth patients ${ }^{1}$. Capacities, such as how to correctly conduct a psychopathological examination or to brush up on verbal and nonverbal communication techniques aimed at producing behavioral changes, can be improved by using multimodal training programs. This could be one of the main conclusions drawn from our study carried out by an ad-hoc multi-disciplinary research team from different institutions, all working at primary care centers ${ }^{2}$. The objective of this study was twofold: a) to determine the effectiveness of an intensive multimodal training program for GPs designed to improve their management of mental-health patients; and b) to ascertain if the program could also be useful to improve the GPs management of their own burnout, job satisfaction and psychological well-being. The results were appealing, although GPs presented heterogeneous results across the different work-related health and well-being parameters analyzed being observed both strengths and weaknesses. Besides, it was not possible to draw strong conclusions because of some methodological limitations of the study mainly related to the small number of participants and that they were not randomly assigned to the treatment conditions.

For a better research contextualization, we would like to mention that this study emerged from a double demand, top-down (primary care center coordinators) and down-top (at the request of the own GP's). In our regular meetings, GPs used to complain about their burnout, job satisfaction and psychological well-being levels. One of the first GPs own request to our Adults Mental Health Service was to tackle this situation; they needed to obtain more and better skills to cope with the increasing number of patients with mental health problems that they were attending. Previous Spanish studies with GPs had already shown worrying levels of GPs' work-related health and psychological well-being ${ }^{3}$, but the situation could have worsened. Giving these circumstances, the primary care coordinators also welcomed any initiative seeking to alleviate this situation. So, initially we carried out a cross-sectional study with $38 \mathrm{GPs}$, analyzing these previously mentioned variables ${ }^{4}$. The results were heterogeneous across the different work-related health parameters analyzed and both strengths and weaknesses were observed. For instance, GPs psychological well-being results were somewhat controversial because the outcomes reflected a high proportion of GPs presenting psychiatric symptoms. They were much more than we expected 
even though the mental state was in our study more thoroughly assessed by a psychiatric clinical interview (as far as we know, for the first time). Taking into account only the cases where mental health support could be somewhat recommended, 21 (55.26\%) GPs suffered from moderate to extremely severe symptoms (anxiety, depression and somatic concerns were predominant). In terms of cooccurring symptoms, 36 (94.74\%) GPs presented more than one symptom at the same time. Among the subgroup with moderate to extremely severe symptoms, 7 (18.42\%) GPs presented more than one symptom simultaneously. So, given these outcomes, we decided to carry out research aimed to study the effectiveness of a multimodal training program to overcome the weaknesses detected. The core purpose of this intervention was to provide GPS with better skills to cope with less personal cost their increased assistance pressure, especially regarding mental health. We didn't intend GPs to become somewhat of a psychiatrist or psychologist, instead of offering some of their key resources and abilities for a better GPs' clinical management of mental health patients.

Beyond the conclusions already exposed in these articles, we would like to mention the significance of several research factors influencing our decision-making. Sometimes, we adopted a position that might be disputed analyzing the related research literature: (a) do not perform the ideal intervention (from a theoretical perspective) but the possible one in a context of real clinical practice, with all its accompanying constraints. In fact, in our case, it was a matter of giving an answer to our service's demand, without additional funding or resources. Although it is questionable and somewhat a balance must be achieved, we support the need to "sacrifice" some degree of internal validity for the ecological validity. Ultimately, we were looking for a real utility for clinical practice. If intervention studies can be placed on a continuum, with a progression from efficacy trials to effectiveness trials, we preferred the last ones for our purposes 5 . (b) we are aware of the scarce reproducibility in social sciences and especially in psychology, although it is a defining feature of science; replication can increase certainty when findings are reproduced and promote innovation when they are not ${ }^{6}$. So, this reproducibility request is also mandatory for our intervention. In this line, we will welcome any initiative in order to replicate our findings, being willing to facilitate all the materials and procedures.

Finally, I would like to comment on the role of the clinical psychology intervention, based on the Integrated Brief Systemic Therapy (IBST), in this multimodal training program (MTP). The MTP was structured as a continuingeducation course with group psychoeducational activities.
The course duration was nine hours in total and consisted of nine weekly sessions. Although all sections were equally important, we have to consider that the vast majority of training sessions were devoted to this ISBT. Besides, at the end of the study, within the ordinary coordinating meetings with GPs, we continued referring to the program's concepts and procedures, mainly regarding psychology. In this context, we would like to take this research as another step towards in examining the IBST' effectiveness both for patients and for GPs, in primary care; the aim is to support IBST's feasibility and usefulness for clinical purposes throughout rooted clinical investigations. Given IBST's pragmatic and empowering perspective with its emphasis on clients' strengths and resources, helping the people to resolve problems quickly and efficiently, it ends us being an appealing tool for clinicians and other mental health professionals. So far, the general results auspices both a potential for IBST in primary care for both collectives ${ }^{7}$. Besides, it is stressed the need to deliver this psychotherapy within a Collaborative Care models (CCMs) framework where the interaction between primary care and mental health providers is structured and multi-disciplinary ${ }^{8}$.

\section{References}

1. Lum AW, Kwok KW, Chong SA. Providing integrated mental health services in the Singapore primary care setting--the general practitioner psychiatric programme experience. Annals of the Academy of Medicine Singapore. 2008; 37(2): 128-131. Retrieved from http://www.ncbi.nlm.nih.gov/pubmed/18327348.

2. Barcons C, García B, Sarri C, et al. Effectiveness of a multimodal training programme to improve general practitioners' burnout, job satisfaction and psychological well-being. BMC Family Practice. 2019; 20(1): 155. https://doi.org/10.1186/s12875-019-1036-2.

3. Matía Cubillo ÁC, Cordero Guevara J, Mediavilla Bravo JJ, et al. Evolución del burnout y variables asociadas en los médicos de atención primaria. Atención Primaria. 2012; 44(9): 532-539. https:// doi.org/10.1016/j.aprim.2010.05.021.

4. Barcons C, García B, Sarri C, et al. Carga actual de los médicos de familia; ¿una necesidad de intervención psicológica? Informaciones Psiquiátricas. 2018; 232(3): 51-67.

5. Singal AG, Higgins PDR, Waljee AK. A primer on effectiveness and efficacy trials. Clinical and Translational Gastroenterology. 2014; 5(1): e45. https://doi.org/10.1038/ctg.2013.13.

6. Open Science Collaboration OS. Estimating the reproducibility of psychological science. Science. 2015; 349(6251): aac4716-aac4716. https://doi.org/10.1126/science.aac4716.

7. Barcons, Carles, Cunillera O, et al. Effectiveness of Brief Systemic Therapy versus Cognitive Behavioral Therapy in routine clinical practice. Psicothema. 2016; 28(3): 298-303. https://doi. org/10.7334/psicothema2015.309.

8. Magaard JL, Liebherz S, Melchior H, et al. Collaborative mental health care program versus a general practitioner program and usual care for treatment of patients with mental or neurological disorders in Germany: protocol of a multiperspective evaluation study. BMC Psychiatry. 2018; 18(1): 347. https://doi.org/10.1186/s12888-0181914-5. 